\title{
P.R4810K, a polymorphism of RNF213, the susceptibility gene for moyamoya disease, is associated with blood pressure
}

\author{
Akio Koizumi - Hatasu Kobayashi - Wanyang Liu - Yukiko Fujii • S. T. M. L. D. Senevirathna • \\ Shanika Nanayakkara · Hiroko Okuda • Toshiaki Hitomi • Kouji H. Harada • \\ Katsunobu Takenaka $\cdot$ Takao Watanabe $\cdot$ Shinichiro Shimbo
}

Received: 20 June 2012 / Accepted: 26 July 2012/Published online: 10 August 2012

(C) The Japanese Society for Hygiene 2012

\begin{abstract}
Background Moyamoya disease-an idiopathic vascular disorder of intracranial arteries-is often accompanied by hypertension. RNF213 has been identified as a susceptibility gene for moyamoya disease. In the present study, the association of p.R4810K (G>A) with blood pressure (BP) was investigated in a Japanese population.

Methodology/principal findings Three independent study populations, the Nyukawa $(n=984)$, Noshiro $(n=2,443)$ and Field $(n=881)$ studies, joined this study. BP, body weight and height were measured. Past and present symptoms and disease and medication histories were assessed by interview. Associations of p.R4810K (rs112735431, ss179362673) of $R N F 213$ with BP were investigated.
\end{abstract}

Electronic supplementary material The online version of this article (doi:10.1007/s12199-012-0299-1) contains supplementary material, which is available to authorized users.

A. Koizumi $(\bowtie) \cdot$ H. Kobayashi · W. Liu · Y. Fujii ·

S. T. M. L. D. Senevirathna - S. Nanayakkara - H. Okuda ·

T. Hitomi - K. H. Harada

Department of Health and Environmental Sciences,

Graduate School of Medicine, Kyoto University, Konoe-cho,

Yoshida, Sakyo-ku, Kyoto 606-8501, Japan

e-mail: koizumi.akio.5v@kyoto-u.ac.jp

K. Takenaka

Department of Neurosurgery,

Takayama Red Cross Hospital, Takayama, Japan

T. Watanabe

Department of Education, Tohoku Bunkyo College,

Yamagata, Japan

S. Shimbo

Department of Food and Nutrition,

Kyoto Women's University, Kyoto, Japan
Two linkage disequilibrium blocks were constructed for moyamoya patients with p.R4810K $(n=140)$ and the general population $(n=384)$ using 39 single nucleotide polymorphisms (SNPs) spanning $390 \mathrm{~kb}$ around RNF213. A total of 60 carriers (3 for AA genotype and 57 for GA genotype) were found in these samples, and the minor allele frequencies were $1.4 \%$ in the Nyukawa and Field studies and $0.2 \%$ in the Noshiro study. Regression analyses adjusted for age, sex and body mass index based on an additive model demonstrated significant associations with systolic BP $(\mathrm{mmHg} /$ allele): $\beta$ (standard error) was 8.2 (2.9) in the Nyukawa study $\left(P=4.7 \times 10^{-3}\right), 18.7(5.4)$ in the Noshiro study $(P=$ $\left.4.6 \times 10^{-4}\right)$ and $8.9(2.0)\left(P=1.0 \times 10^{-5}\right)$ in the three populations. In contrast, diastolic BP showed significant associations only in the Noshiro study. Linkage disequilibrium blocks contained none of the BP-associated proxy SNPs reported by previous studies.

Conclusions/significance Our study suggests that p.R4810K of RNF213 is associated strongly with systolic BP.

Keywords $R N F 213$ - Moyamoya disease - P.R4810K · Systolic blood pressure $\cdot$ Hypertension

\section{Introduction}

Moyamoya disease is an idiopathic occlusive vascular lesion that occurs at the terminal portion of internal carotid arteries in the circle of Willis [1,2]. Familial clustering has been reported for moyamoya disease, with $15 \%$ of cases being reported to have a family history [3]. This familial clustering led to searches for genetic factors, which identified several loci, including 3p24-p26 [4], 6q25 [5], 8q23 [6] and $17 \mathrm{q} 25[7,8]$. The locus on $17 \mathrm{q} 25.3$ was further refined by a linkage analysis [9] and an association study 
[10]. Finally, RNF213 was identified as the susceptibility gene of the $17 \mathrm{q} 25.3$ locus for moyamoya disease [11].

Several polymorphisms specific to Japanese, Korean, Chinese and Caucasian patients with moyamoya disease have been found in $R N F 213$ [11]. Of these, the p.R4810K (rs 112735431, ss179362673; G>A) polymorphism is a founder variant found commonly in Japanese, Korean and Chinese patients. In particular, it is found at rates of $>90 \%$ in Japanese patients and approximately $80 \%$ in Korean patients. More surprisingly, 2-3\% of the Japanese and Korean general populations are carriers of this variant [11]. Given that the prevalence of moyamoya disease in patients is 10.5 per $10^{5}$ people [12] and the rate of carriers of p.R $4810 \mathrm{~K}$ is $3 \%$, only a minor portion of carriers will develop moyamoya disease.

We conducted molecular cloning of a full-length cDNA of RNF213 and found that it comprises 5,207 amino acids. $R N F 213$ has two well-known domains, a RING finger domain and a Walker motif that show ubiquitin ligase activity and ATPase activity, respectively [11]. RNF213 was shown to be a novel functional E3 ligase [11]. Two splicing variants of $R N F 213$ were detected ubiquitously in cDNAs isolated from various types of cells, including vascular endothelial cells [11]. After knockdown of $R N F 213$ in zebrafish embryos, severely abnormal sprouting vessels were seen in the cranial vessels. However, the p.R4810K variant had no apparent detrimental effects on E3 ligase activity.

Moyamoya disease is often accompanied by hypertension $[13,14]$. The pathogenesis of hypertension in patients with moyamoya disease has been attributed to stenosis of the renal arteries, i.e., renovascular hypertension. However, in some cases, hypertension was reported to occur without stenosis of the renal arteries [15]. Such observations suggest that hypertension in moyamoya disease may be caused by a novel mechanism associated with $R N F 213$ variants. If this is the case, it can be hypothesized that hypertension is a phenotype of p.R4810K carriers.

In the present study, we investigated whether p.R4810K elevates blood pressure (BP) in p.R4810K carriers. To examine this possibility, we conducted a genetic epidemiological study in three independent populations.

\section{Methods}

Ethical statement

Ethical approval for the three independent studies was given by the Institutional Review Board and Ethics Committee of Kyoto University School of Medicine, Kyoto University, Japan (Approval number: G140 approval date 10/18/2004). All the subjects provided written informed consent. All the studies involved the use of humans and adhered to the principles of the Declaration of Helsinki and Title 45, US Code of Federal Regulations, Part 46, Protection of Human Subjects.

\section{Study population}

Hypertension was defined as systolic blood pressure (SBP) of $\geq 140 \mathrm{mmHg}$ and/or diastolic blood pressure (DBP) of $\geq 90 \mathrm{mmHg}$ or treatment with antihypertensive medication. The pulse pressure (PP) was calculated as the difference between SBP and DBP.

Samples were donated by participants in three independent studies: the Nyukawa, Noshiro and Field studies. The Nyukawa and Noshiro studies are cohort studies to investigate common diseases. An annual health check-up is provided to the residents as a subsidy program run by the local autonomy at its public health center under the Industrial Safety and Health Act or Elderly Health law. Weight was measured in light clothes on an electrical balance, and height was measured with a stadiometer. Body mass index (BMI) was computed as weight in kilograms divided by the square of the height in meters. BP was measured on the right arm of seated participants by public health nurses after 5 min of rest at each examination using a mercury sphygmomanometer. BP was measured once or more than once when the readings were much higher than past records, i.e., $10 \mathrm{mmHg}$ for SBP and $5 \mathrm{mmHg}$ for DBP. In cases with more than one reading, the later BP reading was used. Clinical data such as past history of medication, past disease history, past and present symptoms, and demographic data (age and birth date) were collected by interview by public health nurses during the clinical examinations. The Nyukawa study started in 2004 in Nyukawa town in Gifu prefecture and has continued to the present, while the Noshiro study started in 1998 in Noshiro city in Akita prefecture and has continued to the present. All participants who underwent health check-ups at local health centers were recruited, and $>95 \%$ of the potential candidates agreed to join the present study. The participants donated blood samples at start of the studies and data collected at the start of the studies were used. Although these two studies are cohort studies, the study design for the present study was a cross-sectional association.

The Field study started in the early 1990s and ended in the mid-1990s as an environmental and nutritional study designed to evaluate exposure to environmental pollutants through dietary routes in nationwide general populations $[16,17]$. Participants were recruited from among farmers by local community farming support stations. More than $90 \%$ of subjects agreed to join the study. Owing to its focus on household information, such as dietary habits and 
sources of vegetables, we selectively recruited more unrelated housewives than males. The researchers visited local towns and collected blood samples. Weight was measured in light clothes on a scale and height was measured with a stadiometer. BP was measured on the right arm of seated participants by physicians after $5 \mathrm{~min}$ of rest at each examination using a mercury sphygmomanometer. $\mathrm{BP}$ readings were measured once, and more than once when readings were much higher (i.e., $10 \mathrm{mmHg}$ for SBP and $5 \mathrm{mmHg}$ for DBP) than the values anticipated by the participants. In cases with more than one reading, the later BP reading was used. Clinical data such as past history of medication, past disease history, past and present symptoms, and demographic data (age and birth date) were collected by interview by physicians during the participant's visit. The samples and data were donated to the Kyoto Specimen sample bank [18, 19]. Since the minor allele frequency (MAF) of p.R4810K in the general population was significantly lower for the Noshiro population $(0.2 \%)$ than for the Nyukawa population $(1.4 \%)$, we selected populations in the western part of Japan in the Field study, i.e., Niigata, Ishikawa, Toyama, Tokyo, Gunma, Nagano, Aichi, Shiga, Kyoto, Kochi, Ehime, Shimane, Yamaguchi and Kagoshima.

We excluded from the analyses subjects with chronic kidney disease, polycystic kidney diseases, diabetic nephropathy and secondary hypertension based on the information obtained by interview. Blood-relatedness of the participants was checked by address, family names and interviews. If there was blood-relatedness among participants, one of the blood-related participants was selected and others were excluded from the analyses. For carriers, interview sheets describing blood-relatedness among carriers within the same communities were re-reviewed. Carriers or related family members were re-interviewed if needed.

Linkage disequilibrium around ss179362673

In the Japanese population, haplotypes carrying p.R $4810 \mathrm{~K}$ in $R N F 213$ were reported to be derived from a common founder [11]. We investigated linkage disequilibrium (LD) blocks in unrelated Japanese controls $(n=384)$ in the Kyoto and Osaka areas in western Japan, and in Japanese patients with p.R4810K and moyamoya disease $(n=140)$ [11]. Thirty-two single nucleotide polymorphisms (SNPs) (rs6565649, rs7216577, rs7406843, rs8078855, rs7217421, rs9902702, rs11869363, rs12451808, rs55996424, rs7222014, rs35968416, rs4890012, rs12150356, rs8070106, rs4889848, rs6565683, rs9913006, rs6565686, rs8065843, rs4074303, rs4890025, rs11869626, rs9898443, rs12601738, rs12185227, rs7502866, rs9911978, rs12950635 rs4890047, rs4889863, rs11655474, rs8080957) were selected on the basis of information in the Hapmap database (http://hapmap.ncbi.nlm.nih.gov/) to cover a region spanning $390 \mathrm{~kb}$ from the $5^{\prime}$ end of $S G S H$ to the $5^{\prime}$ end of Raptor. These 32 SNPs were selected to illustrate the LD blocks using the Tagger program, with criteria of $r^{2}>0.65$ and a MAF of $>0.05$ in Japanese people. Typing for these 32 SNPs plus 7 rare SNPs (ss179362670, ss179362671, ss179362672, ss179362673, ss179362674, ss179362675, ss161110142) was selected. Each LD block was constructed using SNP \& Variation Suite V7. LDs were evaluated by $D^{\prime}$.

\section{Genotyping and quality control}

Genomic DNA was extracted from blood samples with a QIAamp DNA Blood Mini Kit (Qiagen, Tokyo, Japan). Genotyping of the 39 SNPs was conducted using TaqMan probes (TaqMan SNP Genotyping Assays; Applied Biosystems, Tokyo, Japan) using a 7300/7500 Real-Time PCR System (Applied Biosystems, Foster City, CA) as previously reported [11]. Data were cleaned using a quality control process. This process included evaluation of sample and marker call rates, mismatches of sex, age and BP readings, past histories, duplicates and batch effects. Sample relatedness and population stratification were not evaluated genetically. The genotyping results for p.R4810K were confirmed by direct sequencing by the Sanger method in 42 subjects out of 140 patients, as previously reported [11]. The genotype of p.R4810K in two independent genotyping procedures was perfectly matched for control 384 subjects. The call rates for the 39 SNPs were $100 \%$.

\section{Statistical analysis}

Hypertension was analyzed as a binary trait (cases vs controls) using a logistic model under an additive model. Association of p.R4810K with BP was also analyzed by an additive model as a quantitative trait using linear regression models. For subjects taking antihypertensive therapies, BPs were imputed by adding 10 and $5 \mathrm{mmHg}$ to the SBP and DBP values, respectively [20]. Unless otherwise specified, analyses were based on the protocol used in the large studies [21-23]: adjustment for age, sex and BMI: inclusion of subjects taking antihypertensive medications and imputation of their BP. Genetic association analyses were performed using SNP \& Variation Suite V7 (Golden Helix, Bozeman, MT). In the analyses, we tested additive and dominant models. Odds ratio was calculated by the SNP \& Variation Suite for dichotomous phenotypes. HardyWeinberg equilibrium (HWE) was tested in entire populations. The exclusion criteria was $\mathrm{HWE}<10^{-3}$.

A Bonferroni correction of $P=0.05 / 2=0.025$ was applied to the regression analysis. This correction was selected because two parameters, SBP and DBP, were independent, while the other parameters (PP and adjusted 
values) were derivatives of these values. Other statistical analyses were conducted using SAS software (SAS Institute, Cary, NC). A value of $P<0.05$ was considered to indicate statistical significance.

\section{Results}

Demographic characteristics of three populations

The demographic characteristics of the participants in the three studies are summarized in Table 1. The numbers of participants were 984 for the Nyukawa study, 2,443 for the Noshiro study and 881 for the Field study. The percentages of patients taking antihypertensive agents or diuretics ranged from $10.8 \%$ in the Field study to $15.8 \%$ in the Noshiro study. The percentages of patients with hypertension ranged from $12.8 \%$ in the Field study to $17.6 \%$ in the Noshiro study.

The MAFs of p.R4810K were $1.4 \%$ in the Nyukawa and Field studies, and $0.2 \%$ in the Noshiro study (Table 2). The MAF was significantly lower in the Noshiro study than in the Nyukawa and Field studies (Fisher's exact test, $P<1 \times 10^{-5}$ ). Based on interview, none of the carriers had any symptoms related to moyamoya disease or past history of stroke.

Blood-relatedness checked by address and family names did not exclude any subjects in three studies. None of the 60 carriers were confirmed to be blood-related by re-review of the interview sheets.
Table 1 Demographic characteristics of the three samples

Data are shown as values or mean \pm standard deviation (SD)

Table 2 Association of p.R4810K with hypertension by additive model

\begin{tabular}{llll}
\hline Characteristics & Nyukawa study & Noshiro study & Field study \\
\hline Observation period & 2004 -present & 1998 -present & $1990 \mathrm{~s}$ \\
Number of participants & 984 & 2,443 & 881 \\
Men:women & $425: 559$ & $1,229: 1,214$ & $188: 693$ \\
Age (years) & $56.1 \pm 15.1$ & $49.9 \pm 14.0$ & $50.8 \pm 10.4$ \\
Body mass index (BMI) & $22.3 \pm 2.9$ & $23.3 \pm 3.1$ & $23.2 \pm 3.0$ \\
SBP (mmHg) & $125.2 \pm 19.2$ & $126.6 \pm 18.2$ & $129.1 \pm 13.8$ \\
DBP (mmHg) & $72.7 \pm 12.1$ & $77.5 \pm 11.9$ & $76.6 \pm 10.3$ \\
$\%$ Taking antihypertensive or diuretics & 10.9 & 15.8 & 10.8 \\
\% Hypertension & 15.5 & 17.6 & 12.8 \\
\hline
\end{tabular}

\begin{tabular}{|c|c|c|c|c|c|c|c|c|}
\hline \multirow[t]{2}{*}{ Study } & \multicolumn{5}{|c|}{ p.R4810K genotype $(\mathrm{G}>\mathrm{A})$} & & \multicolumn{2}{|c|}{ Dichotomous } \\
\hline & AA & GA & GG & Total & MAF (\%) & & $\mathrm{HT}(+)$ & HT $(-)$ \\
\hline \multirow[t]{5}{*}{ Nyukawa } & 2 & 23 & 959 & 984 & 1.4 & AA & 1 & 1 \\
\hline & & & & & & AG & 6 & 17 \\
\hline & & & & & & GG & 145 & 814 \\
\hline & & & & & & Odds $(95 \%$ CI $)$ & $2.09(0.96$ & $-4.58)$ \\
\hline & HWE & 0.012 & & & & $P$ & 0.08 & \\
\hline \multirow[t]{4}{*}{ Noshiro } & 0 & 11 & 2,432 & 2,443 & 0.2 & AG & 3 & 8 \\
\hline & & & & & & GG & 428 & 2004 \\
\hline & & & & & & Odds $(95 \%$ CI $)$ & $1.76(0.46$ & $-6.64)$ \\
\hline & HWE & 1 & & & & $P$ & 0.368 & \\
\hline \multirow[t]{5}{*}{ Field } & 1 & 23 & 857 & 881 & 1.4 & AA & 0 & 1 \\
\hline & & & & & & AG & 5 & 18 \\
\hline & & & & & & GG & 108 & 749 \\
\hline & & & & & & Odds $(95 \%$ CI $)$ & $1.93(0.70$ & $-5.30)$ \\
\hline & HWE & 0.159 & & & & $P$ & 0.329 & \\
\hline \multirow[t]{5}{*}{ Combined } & 3 & 57 & 4,248 & 4,308 & 0.7 & AA & 1 & 2 \\
\hline & & & & & & AG & 14 & 43 \\
\hline & & & & & & GG & 681 & 3567 \\
\hline & & & & & & Odds (95 \% CI) & \multicolumn{2}{|c|}{$1.71(0.93-3.13)$} \\
\hline & HWE & $1.39 \times$ & $10^{-3}$ & & & $P$ & 0.055 & \\
\hline
\end{tabular}


Table 3 Association of p.R4810K with blood pressures by additive model and dominant model

\begin{tabular}{|c|c|c|c|c|c|c|c|c|c|}
\hline & \multicolumn{3}{|l|}{ SBP } & \multicolumn{3}{|l|}{ DBP } & \multicolumn{3}{|l|}{$\mathrm{PP}$} \\
\hline & $\beta$ & $\mathrm{SE}$ & $p$ & $\beta$ & SE & $p$ & $\beta$ & SE & $p$ \\
\hline \multicolumn{10}{|c|}{ Additive model study } \\
\hline & \multicolumn{9}{|c|}{ Raw data $(\mathrm{mm} \mathrm{Hg})$} \\
\hline Nyukawa & 8.2 & 2.9 & $4.7 \times 10^{-3^{*}}$ & -0.4 & 2.0 & 0.83 & 8.5 & 2.1 & $4.6 \times 10^{-5^{*}}$ \\
\hline Noshiro & 18.7 & 5.4 & $4.6 \times 10^{-4 *}$ & 11.4 & 3.4 & $6.6 \times 10^{-4^{*}}$ & 7.3 & 2.8 & $1.0 \times 10^{-2^{*}}$ \\
\hline Field studies & 4.7 & 2.4 & $5.6 \times 10^{-2}$ & -0.4 & 2.0 & 0.83 & 5.1 & 2.2 & $1.8 \times 10^{-2 *}$ \\
\hline \multirow[t]{2}{*}{ Combined } & 8.9 & 2.0 & $1.0 \times 10^{-5^{*}}$ & 0.6 & 1.9 & 0.70 & 8.3 & 1.3 & $5.4 \times 10^{-11 *}$ \\
\hline & \multicolumn{9}{|c|}{ Adjusted data (mmHg) } \\
\hline Nyukawa & 10.0 & 3.2 & $1.6 \times 10^{-3^{*}}$ & 0.5 & 2.1 & 0.82 & 9.6 & 2.2 & $1.7 \times 10^{-5^{*}}$ \\
\hline Noshiro & 19.1 & 5.8 & $1.0 \times 10^{-3^{*}}$ & 11.6 & 3.6 & $1.2 \times 10^{-3 *}$ & 7.5 & 3.0 & $1.4 \times 10^{-2^{*}}$ \\
\hline Field studies & 6.1 & 2.6 & $2.0 \times 10^{-2^{*}}$ & 0.3 & 2.0 & 0.88 & 5.8 & 2.2 & $8.6 \times 10^{-3^{*}}$ \\
\hline Combined & 10.0 & 2.2 & $5.0 \times 10^{-5^{*}}$ & 1.2 & 1.4 & 0.40 & 8.9 & 1.3 & $2.3 \times 10^{-11^{*}}$ \\
\hline \multicolumn{10}{|c|}{ Dominant model study } \\
\hline & \multicolumn{9}{|c|}{ Raw data $(\mathrm{mm} \mathrm{Hg})$} \\
\hline Nyukawa & 9.2 & 3.2 & $4.4 \times 10^{-3^{*}}$ & -0.2 & 2.2 & 0.94 & 9.4 & 2.4 & $7.6 \times 10^{-5^{*}}$ \\
\hline Noshiro & 18.7 & 5.4 & $4.6 \times 10^{-4^{*}}$ & 11.4 & 3.4 & $6.6 \times 10^{-4^{*}}$ & 7.3 & 2.8 & $1.0 \times 10^{-2^{*}}$ \\
\hline Field studies & 6.3 & 1.7 & $3.0 \times 10^{-4^{*}}$ & 0.04 & 1.4 & 0.97 & 6.2 & 1.5 & $3.1 \times 10^{-5^{*}}$ \\
\hline \multirow[t]{2}{*}{ Combined } & 9.9 & 2.2 & $5.7 \times 10^{-6^{*}}$ & 1.1 & 1.5 & 0.46 & 8.8 & 1.4 & $1.0 \times 10^{-10^{*}}$ \\
\hline & \multicolumn{9}{|c|}{ Adjusted data $(\mathrm{mmHg})$} \\
\hline Nyukawa & 10.8 & 3.5 & $2.3 \times 10^{-3^{*}}$ & 0.6 & 2.3 & 0.78 & 10.2 & 2.5 & $4.0 \times 10^{-5^{*}}$ \\
\hline Noshiro & 19.1 & 5.8 & $1.0 \times 10^{-3^{*}}$ & 11.6 & 3.6 & $1.2 \times 10^{-3^{*}}$ & 7.5 & 3.0 & $1.4 \times 10^{-2^{*}}$ \\
\hline Field studies & 6.7 & 2.8 & $2.0 \times 10^{-2^{*}}$ & 0.5 & 2.2 & 0.82 & 6.9 & 2.5 & $5.4 \times 10^{-3^{*}}$ \\
\hline Combined & 10.8 & 2.4 & $4.9 \times 10^{-6^{*}}$ & 1.5 & 1.5 & 0.92 & 9.2 & 1.4 & $8.5 \times 10^{-11^{*}}$ \\
\hline
\end{tabular}

* Significant association $p<0.025$

Association of p.R4810K with hypertension

The associations of p.R4810K by the additive model for dichotomous traits (hypertension vs normal) were not significant in any of the populations (Table 2).

Regression analyses adjusted for age, sex and BMI revealed significant associations of p.R4810K with SBP and PP (Table 3). Specifically, the $\beta$ [standard error (SE)] values for SBP were 8.2 (2.9) $\mathrm{mmHg}$ /allele for the Nyukawa study and $18.7(5.4) \mathrm{mmHg}$ /allele for the Noshiro study, while those for PP were 8.5 (2.1) $\mathrm{mmHg} /$ allele for the Nyukawa study, 7.3 (2.8) $\mathrm{mmHg}$ /allele for the Noshiro study and 5.1 (2.2) $\mathrm{mmHg} /$ allele for the Field study. The associations of p.R4810K with therapy-adjusted SBP and PP were also confirmed in the three studies. In the three combined populations, p.R4810K was highly associated with SBP [8.9 (2.0) $\mathrm{mmHg} /$ allele] and adjusted SBP [10.0 (2.2) $\mathrm{mmHg}$ /allele]; with PP [8.3 (1.3) $\mathrm{mmHg} / \mathrm{allele}]$ and adjusted PP [8.9 (1.3) mmHg/allele]. The results by additive model are essentially the same by dominant model.

The characteristic features of carriers are shown in Table 4. SBP was significantly higher for the AA + GA genotype than for the GG genotype [136.4 (20.3) vs 126.4
(18.2) $\mathrm{mmHg}$ /allele, $P<0.001$ ], while the male/female ratios, age and BMI did not differ significantly between any two groups. Adjusted SBP (mmHg/allele: mean \pm SD) was 155.3 (19.7) for the AA genotype, 137.8 (22.7) for the GA genotype and 138.7 (22.7) for the AA + GA genotype, which is significantly higher than 127.7 (19.9) with the GG genotype $(P<0.001)$. On the other hand, neither DBP nor adjusted DBP differed between any two groups: the adjusted BP was 78.2 (13.2) and 76.9 (12.5) for GG genotypes, respectively. The \% hypertension was marginally higher in the AA $+\mathrm{AG}$ group than in the GG group (Fisher's exact test, $P=0.06$ ).

Effects on associations of protocol changes for statistical analysis

The present statistical analyses were based on the same protocol [21-23]. The protocol included subjects with antihypertensive medications and adjustment for age, sex and BMI. We investigate the effects of modifications of the protocol on the statistical analyses to determine to what degree the current results are dependent on the protocol. First, we eliminated subjects with antihypertensive 
Table 4 Characteristics of p.R4810K carriers. Values are mean (standard deviation)

\begin{tabular}{llllllll}
\hline Genotype & $N$ & M:F & Age (years) & BMI & & \multicolumn{2}{l}{ Raw blood pressure (mmHg) } \\
& & & & & SBP & DBP \\
\hline AA & 3 & $1: 2$ & $72.7(5.1)$ & $23.2(4.8)$ & $145.3(19.7)$ & $73.3(5.8)$ & 66.7 \\
GA & 57 & $14: 43$ & $51.9(21.4)$ & $23.5(3.9)$ & $135.9(20.4)$ & $77.3(13.1)$ & 24.6 \\
AA + GA & 60 & $15: 45$ & $52.4(14.0)$ & $23.5(3.9)$ & $136.4(20.3) *$ & $77.1(12.8)$ & 25.0 \\
GG & 4,248 & $1,827: 2,421$ & $51.1(13.9)$ & $23.0(3.0)$ & $126.4(18.2)$ & $76.2(11.8)$ & 16.0 \\
\hline
\end{tabular}

* Significantly different from the values in GG genotype $P<0.001$

medications to investigate the effect of genotypes without modifications by medications. Although reduced numbers of subjects decreased statistical power, there were significant associations of p.R4810K with SBP and PP (Table S1). Second, analyses were conducted without adjustment for BMI for the population including subjects with antihypertensive medications. The associations were reproduced without adjustment for BMI (Tables S2 and S3). Changes in the statistical analysis protocol did not alter the results by quantitative regression analyses extensively, suggesting that associations of p.R4810K are robust and reproducible.

\section{LD blocks around p.R4810K of $R N F 213$}

Analyses of the LD blocks of 39 SNPs spanning $390 \mathrm{~kb}$ in patients with p.R4810K and moyamoya disease demonstrated that p.R4810K was in LD with the $3^{\prime}$ half of RNF213 and ENDOV (Fig. 1a). Similar LD blocks were observed in the general control population (Fig. 1b). LD blocks of p.R4810K did not contain any proxy SNPs, which have been reported to be associated with BP in East Asian people or other ethnicities, even when less stringent statistical criteria are applied [21-23].

\section{Discussion}

In the present study, quantitative trait locus analyses demonstrated that p.R4810K was associated significantly with SBP and PP in the general population. The p.R4810K allele resulted in consistently elevated SBP from 4.7 to $18.7 \mathrm{mmHg} /$ allele and PP from 5.1 to $8.5 \mathrm{mmHg} /$ allele. Such large and consistent contributions support our hypothesis that p.R4810K increases SBP.

Several large-scale studies have used the genome-wide association study (GWAS) approach [21-23]. However, none of these studies detected a locus on 17q25.3 for hypertension. Investigations of the LD blocks of p.R4810K failed to reveal any SNPs that have been reported to be associated with BP, even with less stringent $P$ values [21-23]. There is no doubt that recent association studies

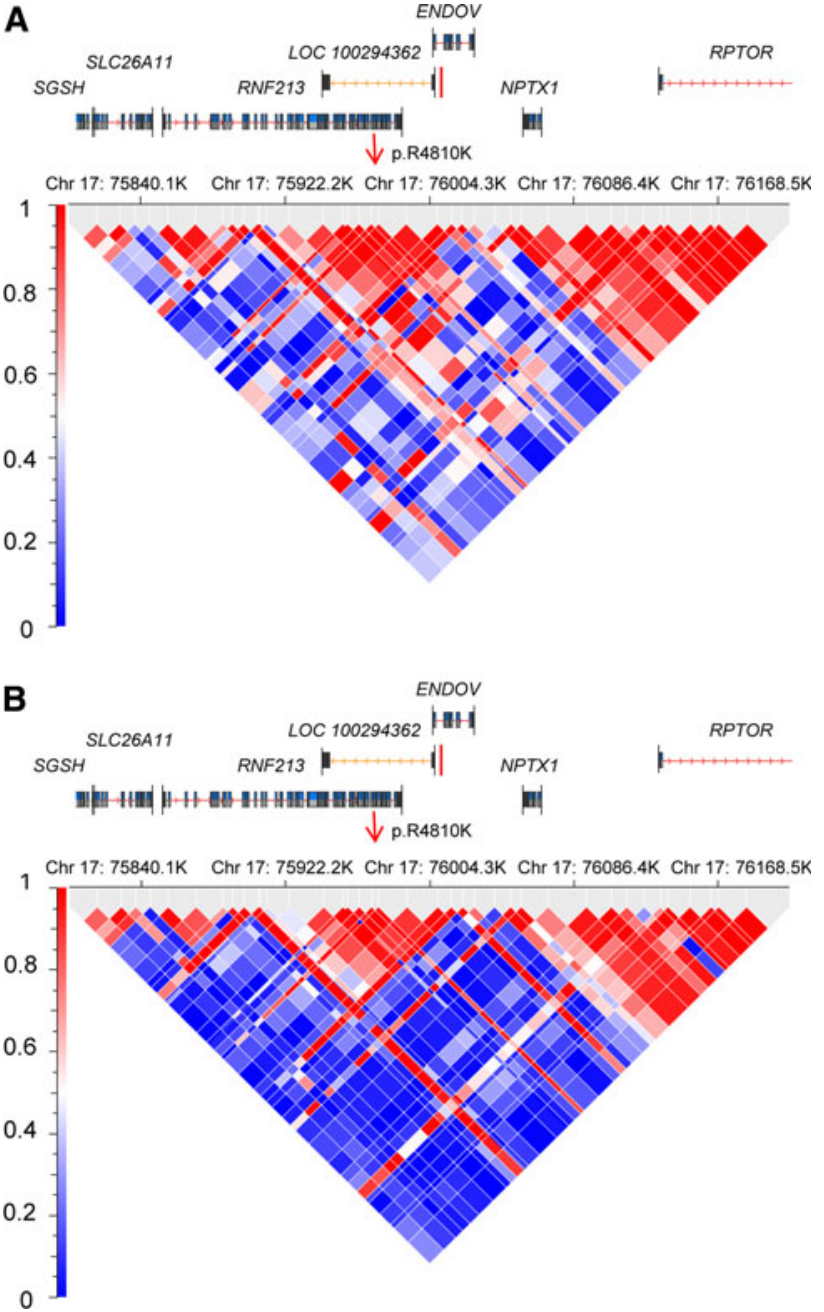

Fig. 1 Linkage disequilibrium (LD) blocks from a region spanning $390 \mathrm{~kb}$ from $S G S H$ to RAPTOR in two independent populations. The LD blocks were constructed with 39 single nucleotide polymorphisms (SNPs). Scales are $D^{\prime}$. The physical positions refer to Genome View Build 36 (http://www.ncbi.nlm.nih.gov/mapview/). a LD blocks for 140 unrelated Japanese patients with p.R4810K and moyamoya disease. b LD blocks for 384 unrelated Japanese subjects

have been successful; however, we consider that there are three major reasons for the failure to detect an association of RNF213 polymorphisms with hypertension. First, the 
critical assumption for the efficient detection of association through GWAS using LD mapping with TagSNPs $\geq 5 \%$ is that, for the susceptibility locus, there is only low level allelic heterogeneity. In the presence of allelic heterogeneities, GWAS with TagSNPs may have missed potentially significant associations [24, 25]. Second, another GWAS assumption is that variants are common. The GWAS protocol usually discards minor alleles, i.e., with allele frequencies smaller than $10 \%$. Thus, minor alleles in RNF213, such as p.R4810K, might have been eliminated from the analysis. Third, even large studies are not free from sample size limitations to detect small population effects even using TagSNPs. For example, a simple genetic power calculation [26] on the assumption (HT prevalence 0.2 ; risk of the allele $=2.0 ; D^{\prime}$ between a tag SNP and risk allele 0.9; tag SNP allele frequency $=0.5)$ reveals that to obtain alpha $=0.05$ and power $=0.80$, more than 50,000 HT Japanese cases would be needed for the low frequencies of risk allele $\sim 0.01$. Given the lower frequencies in the Tohoku areas of Japan, more cases would have been needed. Collectively, although p.R4810K has a discernible influence on $\mathrm{BP}$ in individuals, its small contribution to the population BP makes it extremely difficult to detect, even in large scale GWAS studies.

Given that the current observation can be generalized, carriers of p.R4810K are at risk of high SBP, irrespective of whether they have moyamoya disease. An increase of this magnitude overwhelms the population average of BP treatment effects for single antihypertensive agents [27]. Moreover, it has been suggested that differences in SBP of this magnitude elevate total mortality and the incidence of stroke [28]. Therefore, p.R4810K carriers may need special attention to control their SBP to prevent cerebrovascular events, regardless of whether they are affected by moyamoya disease. In this regard, it remains an enigma why p.R4810K has a strong association with SBP while it does not with DBP. Further studies are warranted to explain the preferential association with SBP.

Hypertension in patients with moyamoya disease has been postulated to be caused by renovascular hypertension $[13,14]$. Similarly, it may occur in carriers without manifestation of moyamoya disease. There may also be other possibilities for the mechanisms of hypertension. For example, it remains to be addressed why hypertension occurs in some patients with moyamoya disease without stenosis of the renal arteries [15]. The physiological role of the p.R $4810 \mathrm{~K}$ variant of $R N F 213$ in the pathological process is unknown. RNF213 comprises a novel class of E3 ligase and is involved in angiogenesis [11]. Therefore, its biochemical and physiological functions need to be elucidated. Further studies are required with a focus on the functional aspects of p.R4810K of RNF213 in SBP.
Except for Down syndrome [29], various disorders associated with moyamoya syndrome are also known to be associated with BP, including Sickle cell disease [30, 31], neurofibromatosis I [32], Noonan syndrome [33, 34], Seckel syndrome [35], familial thoracic aortic aneurysm/ dissection syndrome caused by ACTA2 mutations [36] and $\mathrm{X}$-linked moyamoya syndrome [37]. The genetic association between BP and p.R $4810 \mathrm{~K}$ of $R N F 213$ may not be unique to moyamoya disease, but could instead be common to the pathophysiology associated with moyamoya syndrome. The common pathological processes in these diseases are associated with occlusive vascular lesions. The biomedical mechanisms associated with occlusive vascular lesions may be attributable to endothelial dysfunction, as is the case for prehypertension [38]. Alternatively, it is interesting that patients with ACTA2 mutations have excessive proliferation of vascular smooth muscle cells, and thereby elevated BP [36].

The present study has several limitations, the most major being the population sizes of the carriers of p.R4810K. Although we performed extensive genotyping in the three populations, we could not obtain large numbers of carriers because of the low prevalence of p.R4810K. It is of particular interest that the MAF of p.R4810K is significantly lower in the eastern part of mainland of Japan than in the western part of Japan. It should be investigated in future whether or not the prevalence of moyamoya disease has geographic differences in Japan. Specifically, it may be lower in the eastern part of mainland Japan than in the western part of Japan, as predicted by the low prevalence in Caucasians [11]. In this regard, although we cannot eliminate the effects of population stratification completely, the strength of the present study, namely a single ethnicity, can mitigate such biases. In addition, we did not test genetic relatedness among participants, although we checked it with indirect information. Thus, blood-related samples may be contaminated in associations and lead to inflation of associations. The second limitation of this study is that clinical data were not available for examining renovascular hypertension in carriers. Third, it could be argued p.R4810K is a proxy SNP or that $R N F 213$ is associated directly with BP, and that p.R $4810 \mathrm{~K}$ of $R N F 213$ may elevate BP. At present, we cannot say whether $R N F 213$ is associated with BP, and whether p.R4180K elevates BP directly or not. Further evidence is required to explain the association of p.R4810K with BP biologically. Finally, we cannot exclude a healthy participant effect in the current study. In fact, most of the participants are active both mentally and physically. Handicapped subjects with hypertension-associated cardiovascular diseases or subjects receiving clinical care might have been eliminated in the communities. The present findings may help to determine the pathological consequences of moyamoya disease in 
carriers of p.R4810K. Furthermore, our findings suggest that high SBP may be a common phenotype of carriers of p.R4810K. Finally, although the MAF of p.R4810K in the general population is small, antihypertensive measures may be efficient for this high-risk population.

Acknowledgments We thank Dr. Kayoko Inoue, who died in 2007, Prof. Yasuhiko Wada (University of Kochi Prefecture) and Prof. Masayuki Ikeda (Kyoto Industrial Health Association). We are also grateful to Ms. Misato Umakosi (Kyoto University Graduate School of Medicine). This work was supported mainly by grants from the Ministry of Education, Culture, Sports, Science and Technology of Japan (Kiban Kenkyu A: 22249020) from the ministry of Health, Labour and Welfare of Japan (H23-Nanji-Ippan-019, chaired by Dr. Nobuo Hashimoto) and partially by a grant from Creative Scientific Research (19G50314).

\section{Conflict of interest None.}

\section{References}

1. Takeuchi K, Shimizu K. Hypogenesis of bilateral internal carotid arteries. Brain Nerve. 1957;9:37-43.

2. Suzuki J, Takaku A. Cerebrovascular "moyamoya" disease. Disease showing abnormal net-like vessels in base of brain. Arch Neurol. 1969;20:288-99.

3. Mineharu Y, Takenaka K, Yamakawa H, Inoue K, Ikeda H, et al. Inheritance pattern of familial moyamoya disease: autosomal dominant mode and genomic imprinting. J Neurol Neurosurg Psychiatry. 2006;77:1025-9.

4. Ikeda H, Sasaki T, Yoshimoto T, Fukui M, Arinami T. Mapping of a familial moyamoya disease gene to chromosome 3p24.2-p26. Am J Hum Genet. 1999;64:533-7.

5. Inoue TK, Ikezaki K, Sasazuki T, Matsushima T, Fukui M. Linkage analysis of moyamoya disease on chromosome 6. J Child Neurol. 2000;15:179-82.

6. Sakurai K, Horiuchi Y, Ikeda H, Ikezaki K, Yoshimoto T, et al. A novel susceptibility locus for moyamoya disease on chromosome 8q23. J Hum Genet. 2004;49:278-81.

7. Yamauchi T, Tada M, Houkin K, Tanaka T, Nakamura Y, et al. Linkage of familial moyamoya disease (spontaneous occlusion of the circle of Willis) to chromosome 17q25. Stroke. 2000;31: 930-5.

8. Mineharu $\mathrm{Y}$, Liu $\mathrm{W}$, Inoue $\mathrm{K}$, Matsuura $\mathrm{N}$, Inoue $\mathrm{S}$, et al. Autosomal dominant moyamoya disease maps to chromosome 17q25.3. Neurology. 2008;70:2357-63.

9. Liu W, Hashikata H, Inoue K, Matsuura N, Mineharu Y, et al. A rare Asian founder polymorphism of Raptor may explain the high prevalence of moyamoya disease among East Asians and its low prevalence among Caucasians. Environ Health Prev Med. 2010; 15:94-104.

10. Kamada F, Aoki Y, Narisawa A, Abe Y, Komatsuzaki S, et al. A genome-wide association study identifies RNF213 as the first moyamoya disease gene. J Hum Genet. 2011;56:34-40.

11. Liu W, Morito D, Takashima S, Mineharu Y, Kobayashi H, et al. Identification of RNF213 as a susceptibility gene for moyamoya disease and its possible role in vascular development. PLOS ONE. 2011;6:e22542.

12. Kuroda S, Houkin K. Moyamoya disease: current concepts and future perspectives. Lancet Neurol. 2008;7:1056-66.
13. Yamada I, Himeno Y, Matsushima Y, Shibuya H. Renal artery lesions in patients with moyamoya disease: angiographic findings. Stroke. 2000;31:733-7.

14. Togao O, Mihara F, Yoshiura T, Tanaka A, Kuwabara Y, et al. Prevalence of stenoocclusive lesions in the renal and abdominal arteries in moyamoya disease. AJR Am J Roentgenol. 2004;183:119-22.

15. Limaye CS, Khude S, Pednekar SJ. Moyamoya disease with hypertension in a young adult. J Assoc Physicians India. 2011;59: $124-6$.

16. Shimbo S, Zhang ZW, Moon CS, Watanabe T, Nakatsuka H, et al. Correlation between urine and blood concentrations, and dietary intake of cadmium and lead among women in the general population of Japan. Int Arch Occup Environ Health. 2000;73:163-70.

17. Watanabe T, Shimbo S, Nakatsuka H, Koizumi A, Higashikawa $\mathrm{K}$, et al. Gender-related difference, geographical variation and time trend in dietary cadmium intake in Japan. Sci Total Environ. 2004;329:17-27.

18. Koizumi A, Yoshinaga T, Harada K, Inoue K, Morikawa A, et al. Assessment of human exposure to polychlorinated biphenyls and polybrominated diphenyl ethers in Japan using archived samples from the early 1980s and mid-1990s. Environ Res. 2005;99:31-9.

19. Koizumi A, Harada KH, Inoue K, Hitomi T, Yang HR, et al. Past, present, and future of environmental specimen banks. Environ Health Prev Med. 2009;14:307-18.

20. Cui JS, Hopper JL, Harrap SB. Antihypertensive treatments obscure familial contributions to blood pressure variation. Hypertension. 2003;41:207-10.

21. Levy D, Ehret GB, Rice K, Verwoert GC, Launer LJ, et al. Genome-wide association study of blood pressure and hypertension. Nat Genet. 2009;41:677-87.

22. Ehret GB, Munroe PB, Rice KM, Bochud M, Johnson AD, et al. Genetic variants in novel pathways influence blood pressure and cardiovascular disease risk. Nature. 2011;478:103-9.

23. Kato N, Takeuchi F, Tabara Y, Kelly TN, Go MJ, et al. Metaanalysis of genome-wide association studies identifies common variants associated with blood pressure variation in East Asians. Nat Genet. 2011;43:531-8.

24. Gorlov IP, Gorlova OY, Sunyaev SR, Spitz MR, Amon CI. Shifting paradigm of association studies: value of rare single nucleotide polymorphisms. Am J Human Genet. 2008;82:100-12.

25. Slager SL, Huang J, Vieland VJ. Effect of allelic heterogeneity on the power of the transmission disequilibrium test. Genet Epidemiol. 2000;18:143-56.

26. Purcell S, Cherny SS, Sham PC. Genetic power calculator: design of linkage and association genetic mapping studies of complex traits. Bioinformatics. 2003;19:149-50.

27. Burt VL, Cutler JA, Higgins M, Horan MJ, Labarthe D, et al. Trends in the prevalence, awareness, treatment, and control of hypertension in the adult US population. Data from the health examination surveys, 1960 to 1991 . Hypertension. 1995;26:60-9.

28. Staessen JA, Gasowski J, Wang JG, Thijs L, Den Hond E, et al. Risks of untreated and treated isolated systolic hypertension in the elderly: meta-analysis of outcome trials. Lancet. 2000;355:865-72.

29. Draheim CC, Geijer JR, Dengel DR. Comparison of intimamedia thickness of the carotid artery and cardiovascular disease risk factors in adults with versus without the Down syndrome. Am J Cardiol. 2010;106:1512-6.

30. Koduri PR, Agbemadzo B, Nathan S. Hemoglobin S-C disease revisited: clinical study of 106 adults. Am J Hematol. 2001;68:298-300.

31. Reid HL, Anah CO. Haemorheological parameters in hypertensive Nigerians with and without sickle-cell trait. Angiology. 1985;36:379-86.

32. Zinnamosca L, Petramala L, Cotesta D, Marinelli C, Schina M, et al. Neurofibromatosis type 1 (NF1) and pheochromocytoma: prevalence, clinical and cardiovascular aspects. Arch Dermatol Res. 2011;303:317-25. 
33. Eu-ahsunthornwattana J, Trachoo O, Dejsuphong D, Tunteeratum A, Srichan K, et al. Noonan syndrome, metabolic syndrome and stroke-in-the-young: coincidence, causal or contribution? J Med Assoc Thai. 2010;93:1084-7.

34. Rokicki W, Rokicka A. Noonan syndrome coexisting with essential arterial hypertension in 8 year old boy. Wiad Lek. 2002;55:488-93.

35. Di Bartolomeo R, Polidori G, Piastra M, Viola L, Zampino G, et al. Malignant hypertension and cerebral haemorrhage in Seckel syndrome. Eur J Pediatr. 2003;162:860-2.

36. Guo DC, Papke CL, Tran-Fadulu V, Regalado ES, Avidan N, et al. Mutations in smooth muscle alpha-actin (ACTA2) cause coronary artery disease, stroke, and moyamoya disease, along with thoracic aortic disease. Am J Hum Genet. 2009;84:617-27.

37. Miskinyte S, Butler MG, Herve D, Sarret C, Nicolino M, et al. Loss of BRCC3 deubiquitinating enzyme leads to abnormal angiogenesis and is associated with syndromic moyamoya. Am J Hum Genet. 2011;88:718-28.

38. Giannotti G, Doerries C, Mocharla PS, Mueller MF, Bahlmann $\mathrm{FH}$, et al. Impaired endothelial repair capacity of early endothelial progenitor cells in prehypertension: relation to endothelial dysfunction. Hypertension. 2010;55:1389-97. 